\title{
Decoupling bulk and interfacial contributions to performance in localized high concentration electrolytes for $\mathrm{Li}$ metal batteries
}

\author{
Richard May, ${ }^{\mathrm{a}}$ Julia C. Hestenes, ${ }^{\mathrm{b}}$ and Lauren E. Marbella*a \\ a Department of Chemical Engineering, Columbia University, New York, New York 10027 \\ bepartment of Applied Physics and Applied Mathematics, Columbia University, New York, \\ New York 10027
}

\begin{abstract}
Localized high concentration electrolytes (LHCEs) are a promising class of electrolytes to enable stable cycling of the lithium metal anode. Here, we report the use of operando nuclear magnetic resonance (NMR) spectroscopy to observe electrolyte decomposition during Li stripping/plating and identify the influence of individual components in LHCEs on Li metal battery performance. Data from operando ${ }^{19} \mathrm{~F}$ solution NMR indicates that both bis(fluorosulfonyl)imide (FSI ${ }^{-}$) salt and bis(2,2,2-trifluoroethyl)ether (BTFE) diluent molecules play a key role in solid electrolyte interphase (SEI) formation, in contrast to prior reports that suggest diluents are inert. Using a combination of solution ${ }^{17} \mathrm{O}$ NMR and cyclic voltammetry $(\mathrm{CV})$, we assess differences in solvation and electrochemical reduction in LHCEs and compare to low concentration electrolytes (LCEs). We find that BTFE diluents are chemically (rather than electrochemically) reduced during Li metal battery operation, which can be detected with operando NMR, but not conventional electrochemical methods. Solid-state NMR (SSNMR) and X-ray photoelectron spectroscopy (XPS) measurements confirm that LHCEs decompose to form a SEI on Li metal that contains organic BTFE reduction products as well as high quantities of lithium fluoride from both BTFE and $\mathrm{FSI}^{-}$reduction. Insight into the (electro)chemical reduction mechanisms underpinning SEI formation in LHCEs suggests that fluorinated ethers exhibit tunable reactivity that can be leveraged to control Li deposition behavior.
\end{abstract}

\section{Introduction}

Li metal anodes react with liquid electrolytes to generate a solid electrolyte interphase (SEI) that dictates the stability of Li stripping and plating during battery operation. ${ }^{1-5}$ Increasing Li salt concentration in the electrolyte is a straightforward way to produce smooth Li deposits and high Coulombic efficiency (CE) values $^{6-10}$ to potentially realize high performance Li metal batteries. A larger ratio of Li salt to solvent results in a deficit of solvent molecules that can no longer fully coordinate $\mathrm{Li}^{+}$cations, leading to the formation of contact-ion pairs. ${ }^{6,11}$ High salt concentration electrolytes (HCEs) alter the energy level of the lowest unoccupied molecular orbital (LUMO) and reduction stability of the electrolyte, eventually forming distinct SEI chemistries. ${ }^{12,13}$ For example, increasing the salt concentration from $\sim 1 \mathrm{M}$ to $\sim 4 \mathrm{M}$ in Li bis(fluorosulfonyl)imide (LiFSI) dissolved in dimethyl carbonate (DMC) shifts the LUMO from DMC to $\mathrm{FSI}^{-} .{ }^{14,15}$ As a result, preferential reduction of $\mathrm{FSI}^{-}$at high salt concentration forms a predominantly inorganic SEI that is believed to stabilize Li stripping/plating processes. ${ }^{9,11,16}$

Unfortunately, HCEs are commercially impractical due to low ionic conductivities (from high viscosities) and high costs associated with increasing the salt content in the electrolyte. In contrast, 
localized high concentration electrolytes (LHCEs) are a new class of electrolytes that use a diluent (typically a fluorinated ether) that does not solvate Li ions, to simultaneously lower cost and viscosity while retaining the desirable solvation structures found at high salt concentration (e.g., contact-ion pairs). ${ }^{17-19}$ The LHCE is reported to form microscopic phases of HCE that exist separately from the bulk

diluent, ${ }^{17,20}$ decoupling the interphasial properties (that are dictated by the immediate environment of $\mathrm{Li}^{+}$) from the bulk properties (which are modulated by the overall electrolyte composition) of the electrolyte. Previous work on LHCEs suggests that highly fluorinated diluent molecules (e.g., bis(2,2,2trifluoroethyl)ether (BTFE) and related structures) are not reduced at the Li metal surface during battery operation. ${ }^{17,18}$ Instead, an inorganic-rich SEI is expected from $\mathrm{FSI}^{-}$reduction based on the similarity in solvation structure between LCHEs and HCEs. Yet, this mechanism only considers electrochemical, and not chemical, reduction pathways, which inadvertently neglects the contribution of fluorinated ethers to Li metal passivation.

Here, we use operando ${ }^{19} \mathrm{~F}$ NMR spectroscopy to show that BTFE diluent molecules in a LHCE (1.2 M LiFSI in 1:2 DMC:BTFE by mole) decompose during Li stripping/plating to increase the quantity of $\mathrm{LiF}$ and fluorinated reduction products in the SEI. We find that BTFE molecules are chemically reduced during electrochemical cycling, which is supported by cyclic voltammetry $(\mathrm{CV})$ measurements and electrolyte solvation structures characterized using ${ }^{17} \mathrm{O}$ NMR. The discovery of BTFE decomposition products in the SEI from post-mortem X-ray photoelectron spectroscopy (XPS) and quantitative solidstate NMR (SSNMR) suggests that BTFE plays an important role in stabilizing the interface along with altering properties of the bulk electrolyte. These findings represent a new way to describe and leverage highly fluorinated ether molecules to produce a robust SEI layer on the surface of reactive anode materials.

\section{Results}

\section{Do diluent molecules in LHCEs decompose during Li stripping/plating?}

To investigate the stability of BTFE during electrochemical cycling, we designed a NMR tube cell (details on the cell can be found in Figures S1, S2) that enabled operando electrochemical NMR experiments of a Li/Cu battery in the BTFE-containing LHCE (Figure 1). In this experiment, we find that the amount of $\mathrm{FSI}^{-}$in the electrolyte solution decreases immediately upon cycling, followed quickly by BTFE decomposition (Figures 1, last panel, and Figure S3d). Both signal intensities appear to plateau around $20 \mathrm{~h}$ at $\sim 77 \%$ consumption for BTFE and $\sim 71 \%$ consumption for $\mathrm{FSI}^{-}$based on their initial intensities (Figure 1, last panel). Decomposition of both the salt and the diluent is correlated with a rapid increase in $\mathrm{CE}$ from $23 \%$ in the first cycle to $78 \%$ in the third cycle, fluctuating between $75 \%$ and $95 \%$ in the remaining 46 cycles. These trends suggest that decomposition of both $\mathrm{FSI}^{-}$and BTFE at the Li metal surface are responsible for forming a SEI layer that promotes high CE and uniform Li stripping/plating. No soluble species from BTFE/FSI ${ }^{-}$reduction are observed during operando NMR nor ex situ NMR (Figure 4b), suggesting that these intermediates are short-lived before deposition in the SEI layer (N.B. NMR experiments are collected every 3 min and transient intermediates may not be captured if they are present at low concentrations). 


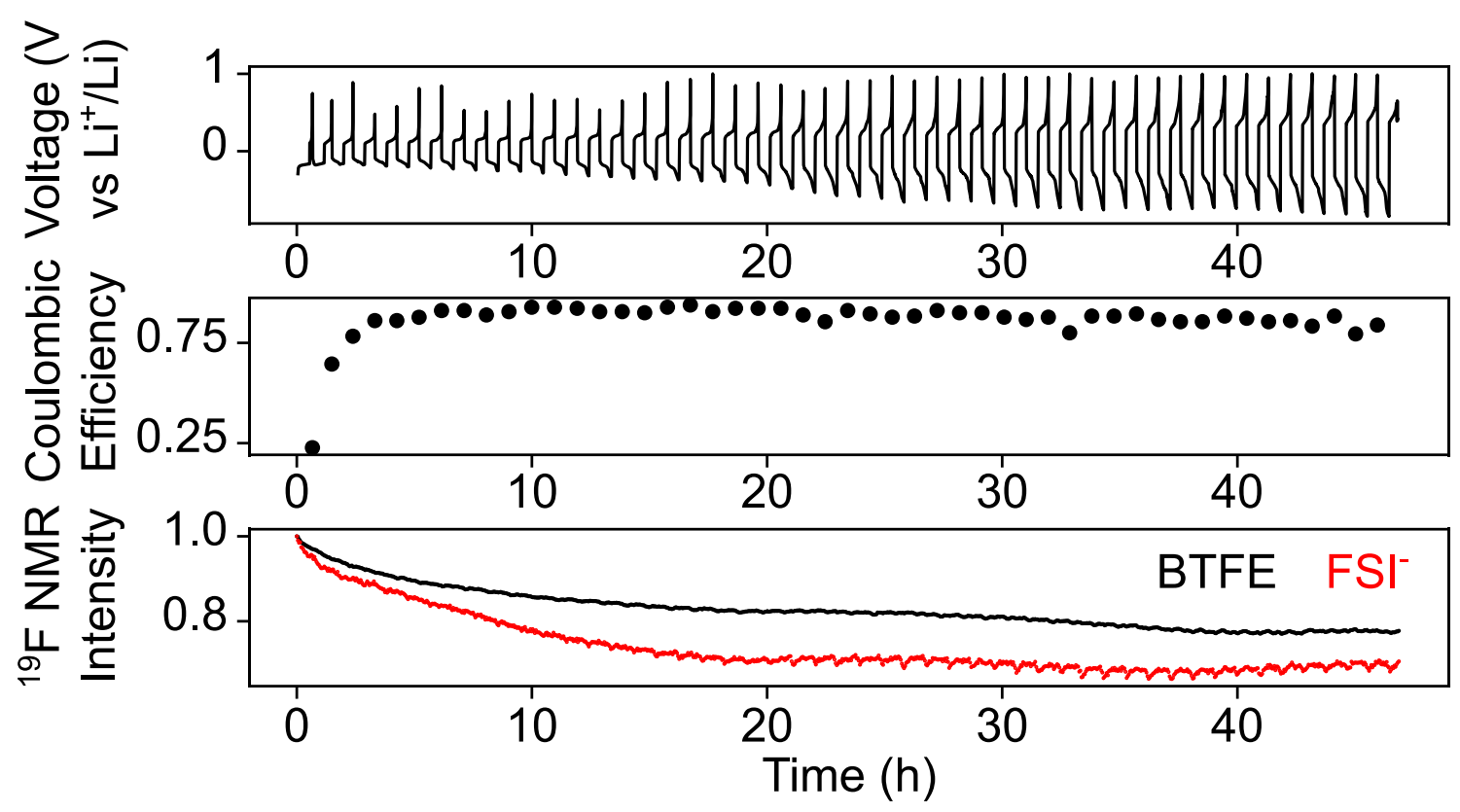

Figure 1. Operando ${ }^{19} \mathrm{~F}$ solution NMR performed while cycling a $\mathrm{Li} / \mathrm{Cu}$ cell in a NMR tube. The cell was cycled for $46.8 \mathrm{~h}$, with each cycle comprising Li plating on $\mathrm{Cu}$ for $30 \mathrm{~min}$ at $2 \mathrm{~mA} \mathrm{~cm}^{-2}$, followed by stripping to $1 \mathrm{~V}$. The top panel shows the voltage profile vs $\mathrm{Li}^{+} / \mathrm{Li}$ as a function of time, and the middle panel shows the $\mathrm{CE}$ for each cycle. The bottom panel shows the normalized ${ }^{19} \mathrm{~F}$ NMR intensity of BTFE (black) and $\mathrm{FSI}^{-}$(red) as a function of time.

\section{Bulk electrolyte solvation structure and expected (electro)chemical reduction pathways}

We performed CV measurements to assess electrolyte decomposition pathways in the LHCE compared to the LCE (Figure 2). An anodic peak at $1.4 \mathrm{~V}$ and a cathodic peak at $0.7 \mathrm{~V}$ are assigned to anodic stripping

a) LCE: 1.2 M LiFSI in DMC

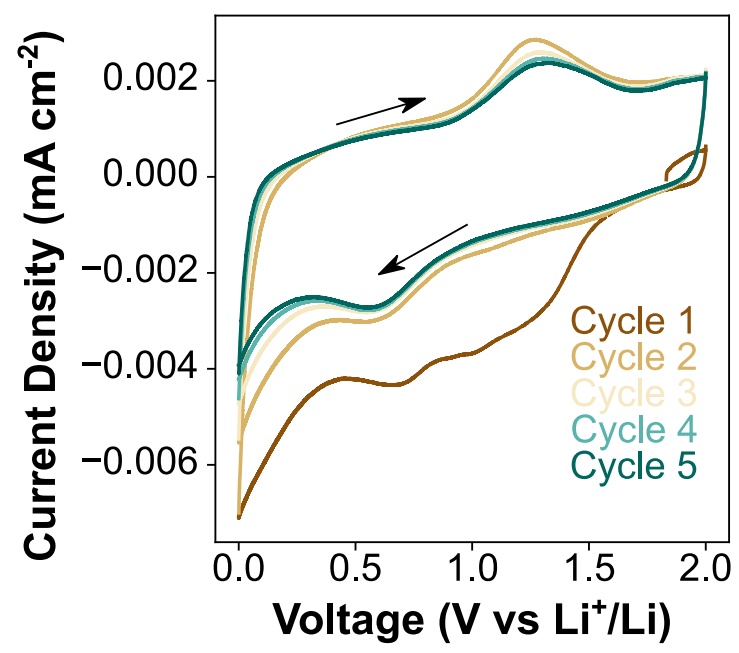

b) LHCE: 1.2 M LiFSI in 1:2 DMC:BTFE

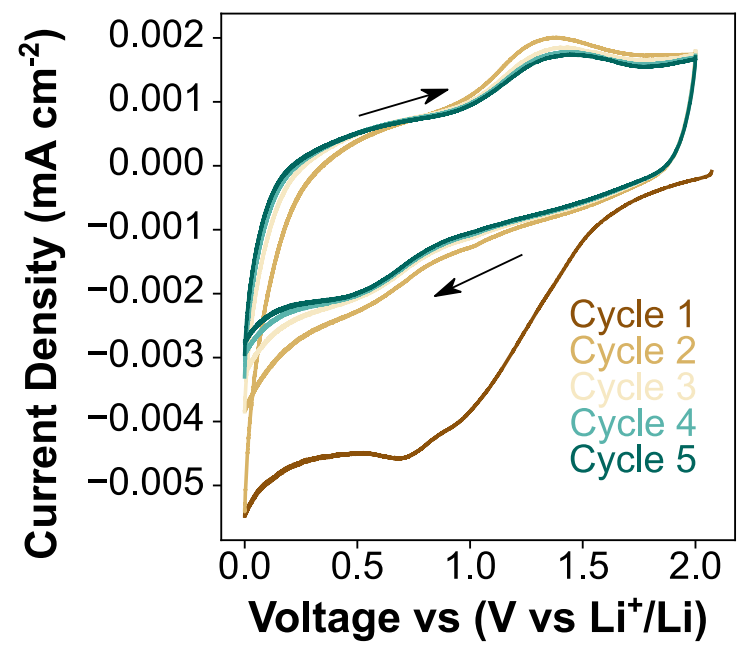

Figure 2. Cyclic voltammograms of Li/stainless steel coin cells in the LCE (a) and the LHCE (b). Each cell underwent 5 cycles from $0-2 \mathrm{~V}$ vs $\mathrm{Li}^{+} / \mathrm{Li}$ at $0.1 \mathrm{mV} / \mathrm{s}$. 
of $\mathrm{Li}$ and underpotential deposition, respectively. ${ }^{21}$ In the first cycle, the onset of a cathodic peak at $1.4 \mathrm{~V}$ likely corresponds to DMC reduction, ${ }^{22}$ and a separate cathodic peak at $1.0 \mathrm{~V}$ corresponds to $\mathrm{FSI}^{-}$ reduction. ${ }^{23}$ These redox events are likely mitigated over subsequent cycles due to passivation of the $\mathrm{Li}$ surface. The difference between the first cycle voltammogram and the voltammograms of subsequent cycles is much more pronounced in the LHCE than the LCE, showing that electrolyte decomposition slows to a greater extent after the first cycle in the LHCE than in the LCE. Thus, addition of BTFE results in fast initial formation of the SEI that is more stable compared to the SEI formed in the LCE. Further, there are no obvious peaks which can be assigned to BTFE reduction, suggesting that the decomposition of BTFE does not occur electrochemically.

To examine the effects of diluent addition on the solvation environment of $\mathrm{Li}^{+}$, we performed ${ }^{17} \mathrm{O}$ NMR on the LCE and LHCE, as well as their respective solvents (Figure 3). Solution ${ }^{17} \mathrm{O}$ NMR chemical shifts and linewidths can be used to describe and quantify ion solvation structures because oxygen atoms on carbonate and ether molecules coordinate to $\mathrm{Li}^{+}$in solution ${ }^{24}$ and ultimately dictate reduction potential. Changes in both the chemical shift and full width at half maximum (FWHM) of ${ }^{17} \mathrm{O}$ signals upon Li salt addition arise due to averaging of the ${ }^{17} \mathrm{O}$ NMR resonances of "free" ${ }^{17} \mathrm{O}$ sites (not interacting with $\mathrm{Li}^{+}$) with the corresponding "bound" ${ }^{17} \mathrm{O}$ sites (coordinated to $\mathrm{Li}^{+}$). Thus, oxygen sites that coordinate strongly with $\mathrm{Li}^{+}$will exhibit low frequency ${ }^{17} \mathrm{O}$ chemical shifts due to increased shielding, and may experience an increase in FWHM due to lower mobility (note that larger FWHM values may also arise from increased viscosity and slower tumbling). Conversely, oxygen sites that interact weakly or negligibly with $\mathrm{Li}^{+}$may
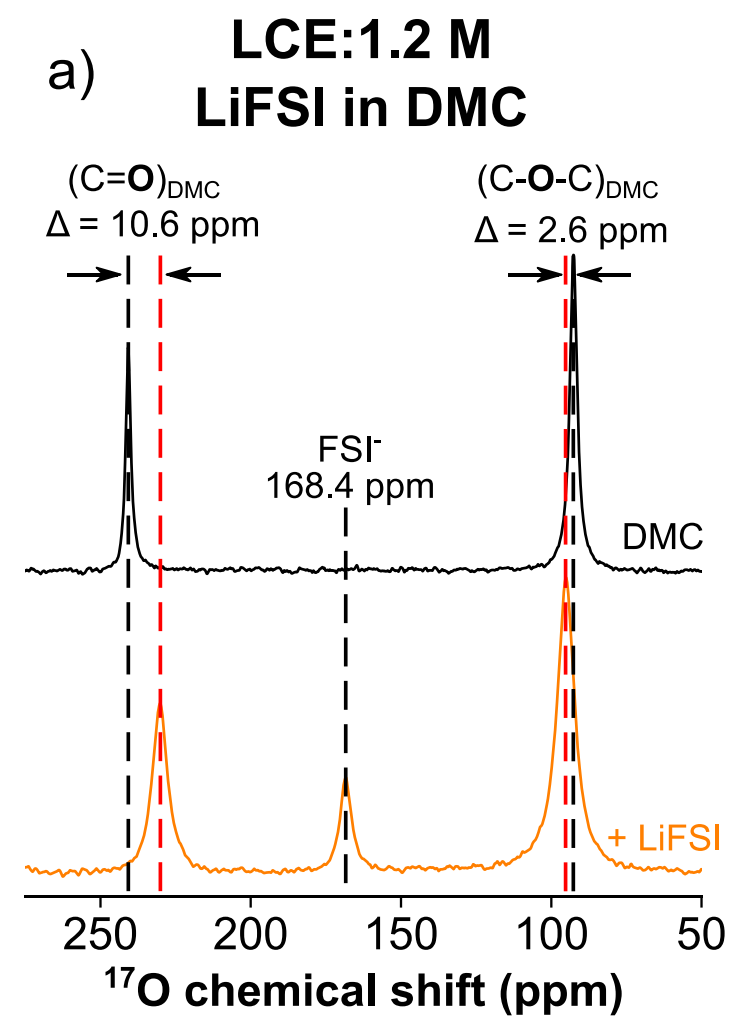
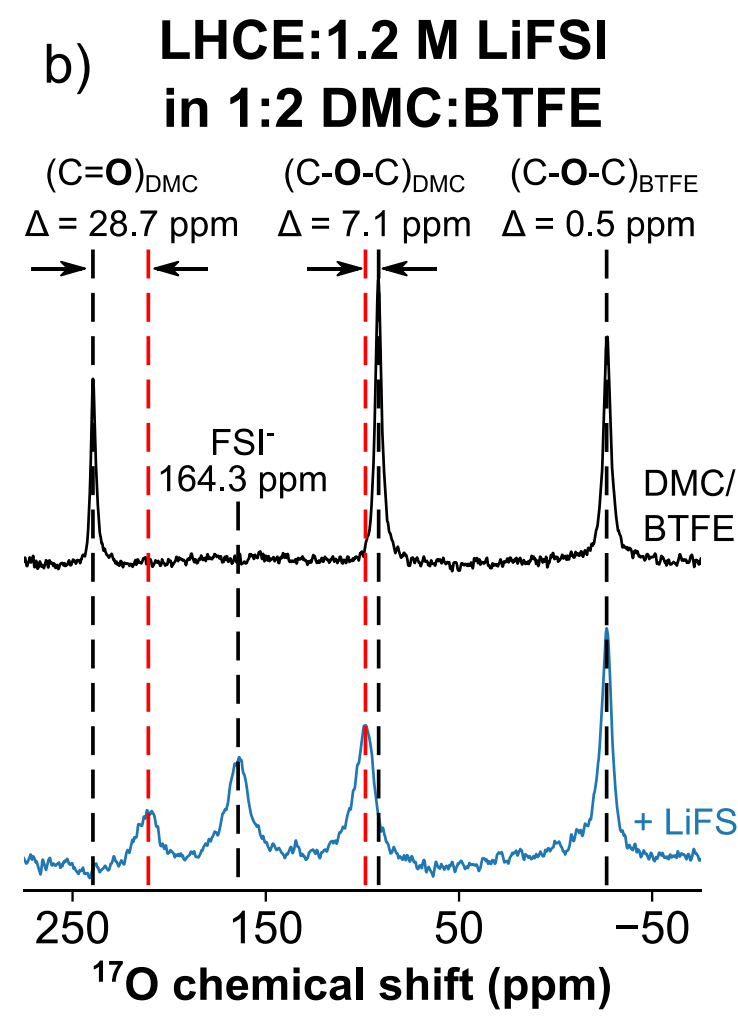

Figure 3. ${ }^{17} \mathrm{O}$ NMR spectra for the (a) LCE (orange, bottom spectrum) and (b) LHCE (blue, bottom spectrum), as well as their respective solvents (DMC and 1:2 DMC:BTFE by mole are both shown in black at the top of each stack). Peak assignments are shown using dashed lines for the carbonyl oxygen and ethereal oxygen atoms on DMC as well as BTFE and $\mathrm{FSI}^{-}$. Changes in chemical shifts $(\Delta)$ are denoted with arrows. 
Table 1. ${ }^{17} \mathrm{O}$ NMR shifts and FWHM values from Lorentzian fits of the data for different electrolytes shown in Figure 3.

\begin{tabular}{|c|c|c|c|c|c|c|c|c|}
\hline & \multicolumn{4}{|c|}{${ }^{17} \mathrm{O}$ NMR shifts } & \multicolumn{4}{|c|}{${ }^{17} \mathrm{O}$ NMR FWHM } \\
\hline $\begin{array}{l}\text { Electrolyte } \\
\text { composition }\end{array}$ & $\begin{array}{c}(\mathrm{C}=\mathrm{O})_{\text {DMC }} \\
{ }^{17} \mathrm{O} \text { shift } \\
(\mathrm{ppm})\end{array}$ & $\begin{array}{c}\text { FSI }^{-17} \mathbf{O} \\
\text { shift } \\
(\mathbf{p p m})\end{array}$ & $\begin{array}{c}(\mathrm{C}- \\
\text { O) } \\
\text { DMC } \\
{ }^{17} \text { O shift } \\
\text { (ppm) }\end{array}$ & $\begin{array}{c}\text { BTFE } \\
{ }^{17} \text { O shift } \\
\text { (ppm) }\end{array}$ & $\begin{array}{c}(\mathrm{C}=\mathrm{O})_{\mathrm{D}} \\
{ }^{\mathrm{MC}}{ }^{17} \mathrm{O} \\
\text { FWHM } \\
(\mathrm{Hz})\end{array}$ & $\begin{array}{c}\text { FSI }^{-17} \mathbf{O} \\
\text { FWHM } \\
(\mathbf{H z})\end{array}$ & $\begin{array}{c}\mathrm{C}- \\
\text { O) } \\
{ }^{17} \mathrm{OMC} \\
\text { FWHM } \\
(\mathbf{H z})\end{array}$ & $\begin{array}{c}\text { BTFE } \\
{ }^{17} \mathrm{O} \\
\text { FWHM } \\
(\mathrm{Hz})\end{array}$ \\
\hline DMC & 240.8 & & 92.6 & & 109.4 & 0.0 & 153.7 & \\
\hline $\begin{array}{l}\text { 1.2 M LiFSI } \\
\text { in DMC }\end{array}$ & 230.2 & 168.4 & 95.2 & & 296.7 & 222.9 & 359.6 & \\
\hline $\begin{array}{l}2: 1 \\
\text { BTFE:DMC }\end{array}$ & 239.4 & & 91.7 & -26.7 & 142.7 & & 196.3 & 240.1 \\
\hline $\begin{array}{l}\text { 1.2 M LiFSI } \\
\text { in 2:1 } \\
\text { BTFE:DMC }\end{array}$ & 210.7 & 164.3 & 98.8 & -26.2 & 461.7 & 642.9 & 535.1 & 361.8 \\
\hline
\end{tabular}

exhibit no change or even a higher frequency ${ }^{17} \mathrm{O}$ chemical shift due to deshielding, and should experience smaller changes in FWHM.

After addition of 1.2 M LiFSI to DMC (LCE, Figure 3a), we observe strong interaction between the DMC carbonyl oxygen and $\mathrm{Li}^{+}$, which manifests as a $\sim 9 \mathrm{ppm}$ shift to lower frequencies and a $171 \%$ increase in FWHM in ${ }^{17} \mathrm{O}$ NMR (Table 1). The ethereal oxygen in DMC (Figure 3a) exhibits a smaller high frequency shift of $\sim 2.5 \mathrm{ppm}$, consistent with increased deshielding, as well as a $134 \%$ increase in FWHM. In the LHCE (Figure 3b), we observe larger changes in the ${ }^{17} \mathrm{O}$ NMR signatures for DMC as compared to the LCE. The ${ }^{17} \mathrm{O}$ NMR resonances that correspond to the carbonyl oxygens in DMC exhibit a shift of $\sim 29$ ppm to lower frequency that is concomitant with a $224 \%$ increase in FWHM. Likewise, the ethereal oxygen atoms in DMC show greater change upon salt addition in the LHCE than in the LCE, with a shift to higher frequency of $\sim 7 \mathrm{ppm}$ and a $173 \%$ increase in FWHM. The difference in ${ }^{17} \mathrm{O}$ chemical shift of the BTFE diluent upon salt addition is minimal, with a shift to higher frequency of 0.4 ppm, indicating that BTFE does not interact with $\mathrm{Li}^{+}$. The increased FWHM of the BTFE resonance in ${ }^{17} \mathrm{O}$ NMR upon addition of the LiFSI is consistent with an increase in dynamic viscosity (from $1.78 \mathrm{cP}$ to $1.84 \mathrm{cP}$, Table S1) and a corresponding decrease in ionic diffusion coefficients measured in pulsed field gradient (PFG) NMR (Figures S4-S7, Table S2). These data suggest that the change in ${ }^{17} \mathrm{O}$ FWHM for BTFE is due to slower tumbling in the bulk electrolyte when LiFSI is present and not from interaction with ions in solution.

Taken together, $\mathrm{CV}$ and solution ${ }^{17} \mathrm{O}$ NMR are consistent with findings from computational models that suggest favorable electrochemical reduction of $\mathrm{FSI}^{-}$over DMC or BTFE in LHCEs. ${ }^{15}$ To reconcile the lack of electrochemical reduction of BTFE with the obvious BTFE decomposition observed in operando ${ }^{19} \mathrm{~F}$ NMR, we next examined the resulting SEI compositions post-mortem.

\section{What is the resulting SEI structure and how does it alter Li deposition behavior?}


Quantitative ${ }^{19} \mathrm{~F}$ solution NMR shows little $\mathrm{FSI}^{-}$fragmentation after Li metal anodes are cycled in the LHCE (Figure 4b). In contrast, cycling in the LCE (without BTFE) leads to $76 \%$ more FSI $^{-}$ decomposition as indicated by ${ }^{19} \mathrm{~F}$ resonances at 51.5 and $50.2 \mathrm{ppm}$ that may correspond to soluble $\mathrm{SO}_{2} \mathrm{NSO}_{2} \mathrm{~F}^{-}$type structures observed in molecular dynamics simulations. ${ }^{25} \mathrm{Li}$ SSNMR measurements conducted on the corresponding Li electrodes cycled in the LCE also show that $\mathrm{Li}_{\mathrm{x}} \mathrm{S}_{\mathrm{y}}$ species are observed in the SEI (Figure S8 shows a characteristic shift for Li polysulfides at $7 \mathrm{ppm}$ ) when BTFE is not present to mitigate the $\mathrm{FSI}^{-}$fragmentation seen in ${ }^{19} \mathrm{~F}$ solution NMR. S 2 p XPS shows very low quantities ( $1 \%$ of the sample, Figure S9 and Table $\mathrm{S} 3$ ) of $\mathrm{Li}_{2} \mathrm{~S}$-type compounds in the SEI after cycling in the LCE, which is not expected based on SSNMR measurements where the quantities of $\mathrm{Li}_{\mathrm{x}} \mathrm{S}_{\mathrm{y}}$ are approximately equal to other Li-containing compounds in the SEI (Figure S8). We believe that this discrepancy arises because soluble $\mathrm{Li}_{\mathrm{x}} \mathrm{S}_{\mathrm{y}}$ structures are rinsed away during XPS sample preparation, whereas SSNMR measurements do not require sample washing prior to analysis. Extensive literature from the Li-sulfur community suggests that $\mathrm{Li}_{\mathrm{x}} \mathrm{S}_{\mathrm{y}}$ compounds lead to performance degradation in $\mathrm{Li}$ metal batteries, ${ }^{26,27}$ possibly contributing the poor performance of Li metal anodes in LCEs containing $\mathrm{FSI}^{-}$salts.

Ex situ ${ }^{19}$ F SSNMR and F 1s XPS characterization of the fluorinated SEI on Li metal indicates that more LiF is present in the SEI on Li metal anodes cycled in the LHCE vs in the LCE (Figure 4c

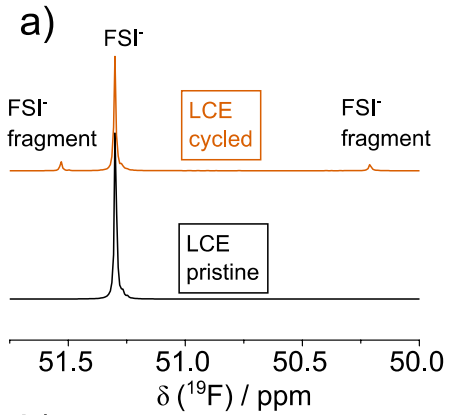

b)

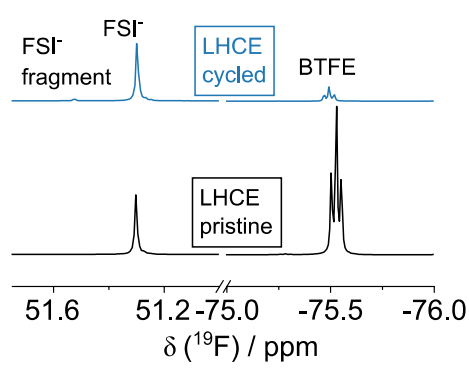

c)

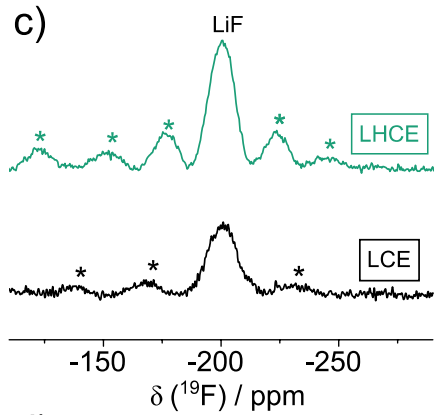

d)

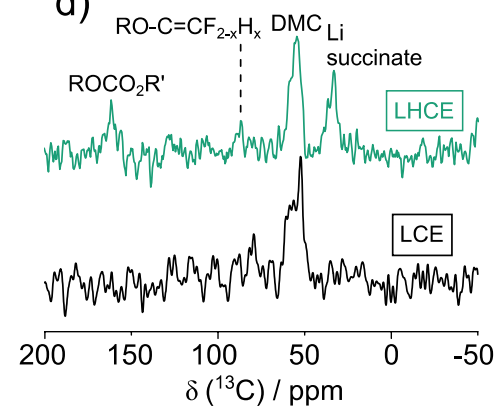

e) LCE: $1.2 \mathrm{M}$ LiFSI $D M C$
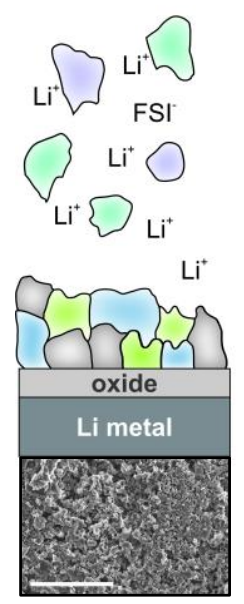

$\bigcirc \mathrm{Li}_{2} \mathrm{O} \bigcirc$ carbonates

LiF $\mathrm{FSI}^{-}$fragments

$\mathrm{Li}_{x} \mathrm{~S}_{y}$ organofluorides
LHCE: 1.2 M LIFSI DMC:BTFE

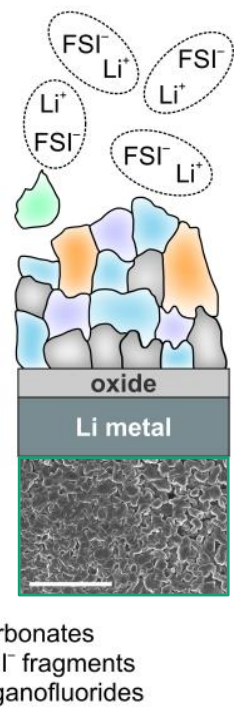

Figure 4. a) ${ }^{19} \mathrm{~F}$ solution NMR of pristine LCE (black) and LCE after 48 galvanostatic $2 \mathrm{~h}$ cycles at $1 \mathrm{~mA} / \mathrm{cm}^{2}$ (orange) in Li/Li symmetrical cells. b) ${ }^{19} \mathrm{~F}$ solution NMR of pristine LHCE (black) and LHCE after 48 galvanostatic $2 \mathrm{~h}$ cycles at $1 \mathrm{~mA} / \mathrm{cm}^{2}$ (blue) in Li/Li symmetrical cells. c) ${ }^{19} \mathrm{~F}$ SSNMR of Li electrodes after 30 galvanostatic $2 \mathrm{~h}$ cycles at $1 \mathrm{~mA} / \mathrm{cm}^{2}$ in the LCE (black) and LHCE (green) in Li/Li symmetrical cells. d) ${ }^{13} \mathrm{C}$ SSNMR of Li electrodes extracted from Li/Li symmetrical cells after 30 galvanostatic $2 \mathrm{~h}$ cycles at $1 \mathrm{~mA} / \mathrm{cm}^{2}$ in the LCE (black) and LHCE (green). Samples were spun at $12.5 \mathrm{kHz}$ MAS frequency for the LHCE sample and $18 \mathrm{kHz}$ MAS frequency for the LCE sample for both ${ }^{13} \mathrm{C}$ and ${ }^{19} \mathrm{~F}$ measurements. e) Cartoon depicting the differences in SEI composition between the LHCE and LCE with representative SEMs showing microstructural morphology after plating (scale bar $=30 \mu \mathrm{m}$ ).

shows $80 \%$ more LiF based on integration of quantitative ${ }^{19} \mathrm{~F}$ SSNMR, Figure S9 shows the corresponding XPS). Higher quantities of LiF in the SEI, combined with the BTFE decomposition 
observed in operando and ex situ ${ }^{19} \mathrm{~F}$ NMR for LHCEs, provides compelling evidence that BTFE reduction contributes to LiF formation in the SEI.

Mitigating the formation of soluble organic decomposition products in favor of surface-stable organic species in the LHCE likely improves SEI stability and Li deposition behavior. ${ }^{28,29}{ }^{1} \mathrm{H}$ and ${ }^{13} \mathrm{C}$ solution NMR of the cycled LHCE shows no soluble organic decomposition products (Figures S11, S12), whereas cycled LCEs exhibit a variety of soluble species in ${ }^{1} \mathrm{H}$ (Figure S10) and ${ }^{19} \mathrm{~F}$ (Figure 4a, b) solution NMR, including lithium methyl carbonate (LMC) and ethyl methyl carbonate (EMC). Figure S10 shows the complete NMR assignments for organic decomposition products. Meanwhile, ${ }^{13} \mathrm{C}$ SSNMR showed a more heterogeneous distribution of organic decomposition products from the LHCE than the LCE, with polycarbonates (161.5 ppm), fluorocarbons $(86.6 \mathrm{ppm}),{ }^{30} \mathrm{Li}$ succinate $(34.3 \mathrm{ppm}),{ }^{31}$ and DMC (55.6 ppm) observed, whereas only residual solvent was observed in the LCE (Figure 4d). These results are consistent with $\mathrm{C} 1 \mathrm{~s}$ XPS analyses, which indicate that $\mathrm{C}-\mathrm{O}$ and $\mathrm{C}=\mathrm{O}$ environments are present in both the LCE and LHCE (Figure S9). Although we detect fluorinated C=C species in our ${ }^{13} \mathrm{C}$ SSNMR, we do not observe unsaturated fluorocarbons in either the ${ }^{19} \mathrm{~F}$ SSNMR or the C 1s XPS (Figures 4a and S16). While small amounts of fluorocarbons are, in principle, detectable using ${ }^{19} \mathrm{~F}$ SSNMR, they likely overlap with the strong sideband pattern observed for LiF in the SEI (marked with asterisks in Figure 4c) and may be removed during rinsing of Li electrodes prior to XPS analysis. Alternatively, these compounds may be completely defluorinated during BTFE reduction to generate $\mathrm{C}=\mathrm{C}$ groups with no appended $\mathrm{F}$ and $\mathrm{LiF}$.

Overall, the SEI formed in the LCE is composed of soluble inorganic FSI- fragments and alkyl carbonates as well as $\mathrm{Li}_{\mathrm{x}} \mathrm{S}_{\mathrm{y}}$ and $\mathrm{LiF}$ (Figure 4e). Conversely, the LHCE shows almost no detectable soluble decomposition products after cycling, consistent with improved Li passivation in the presence of BTFE. ${ }^{28,29}$ The SEI generated on the surface of Li metal after cycling in the LHCE contains higher quantities of $\mathrm{LiF}$, polycarbonates/alkyl carbonates, and unsaturated organic structures. This LiF-rich, chemically diverse SEI formed in the LHCE is correlated with smooth Li plating behavior observed in scanning electron micrographs (SEM, Figure 4e) as well as high CE (Figure S13; 98.2\% over 100 cycles for the LHCE vs $17.4 \%$ for the LCE) and a more stable overpotential during galvanostatic cycling of $\mathrm{Li} / \mathrm{Li}$ symmetric coin cells (Figure S14). These results suggest that changes in SEI chemistry brought about by diluent decomposition are responsible for improvements in both Li deposition and electrochemical performance in Li metal batteries.

\section{Discussion}

Operando NMR, CV, and post-mortem compositional analysis of electrolyte decomposition pathways strongly suggest that the diluent molecules present in LHCEs participate in chemical reduction and SEI formation. Reduction of the BTFE diluent is correlated with smooth Li deposition morphologies and high $\mathrm{CE}$ values, leading us to reevaluate the role of highly fluorinated ether molecules in Li metal battery performance. Instead of acting as inert "filler" molecules which serve only to alter bulk electrolyte solvation structures, BTFE contributes to SEI formation, and as such, can be leveraged to tune SEI compositions and alter subsequent Li deposition behavior. In BTFE, chemical reduction involves elimination at the $\mathrm{C}-\mathrm{F}$ bond to form $\mathrm{LiF}$ and $\mathrm{C}=\mathrm{C}$ species, which is consistent with chemical defluorination models proposed for tris(2,2,2-trifluoroethyl)orthoformate (TFEO) by Balbuena and coworkers, ${ }^{14}$ and suggests that other diluent decomposition reactions proceed in a similar fashion. SSNMR characterization of the resulting SEI shows that BTFE-containing electrolytes generate large quantities of $\mathrm{LiF}$ as well as $\mathrm{RO}-\mathrm{C}=\mathrm{CF}_{2-\mathrm{x}} \mathrm{H}_{\mathrm{x}}$ moieties in the SEI. Similar unsaturated organic and inorganic compounds are observed during cycling of fluoroethylene carbonate (FEC)-containing electrolytes, where 
FEC is electrochemically defluorinated. ${ }^{32,33}$ The similarities between BTFE and FEC product outcomes suggests that either chemical or electrochemical mechanisms can be used to deposit LiF and unsaturated organic structures in the SEI. We expect that the tunable chemistry of small molecules can be leveraged to further alter the reactivity of fluorinated additives, offering a new route to control Li deposition behavior.

The formation of a passivating SEI layer on Li metal is critical to achieving smooth Li deposition morphologies. ${ }^{28,29,34-36}$ Importantly, we see that the SEI produced during Li stripping/plating in the LHCE protects the electrolyte from the Li metal surface and prevents $\mathrm{FSI}^{-}$and $\mathrm{DMC}^{-}$fragmentation. Soluble $\mathrm{FSI}^{-}$ and DMC reduction products do not adhere to the Li metal surface and cannot stabilize the SEI, resulting in high surface area Li deposits and low CE values. Shuttling of soluble polysulfide species generated in LCE during FSI $^{-}$breakdown likely also contribute to poor battery performance and is a commonly cited issue in the Li-S battery literature. ${ }^{26,27,37}$ Our data indicates that BTFE diluent addition suppresses the FSI decomposition pathways that produce soluble polysulfides and other fragments in favor of more stable degradation products, such as $\mathrm{LiF}$ and unsaturated organics, that protect the Li surface from continuous reaction with the electrolyte.

\section{Conclusion}

Operando ${ }^{19} \mathrm{~F}$ NMR measurements were required to pinpoint the chemical decomposition of BTFE, which could not have been achieved using electrochemical methods. The operando NMR cell reported in this work has a simple architecture that can be applied to two-electrode experiments and produces fast, quantitative, chemically accurate measurements during battery operation. Insight from NMR, XPS, and $\mathrm{CV}$ measurements suggest that optimization of Li metal interphasial chemistry is key to enabling high performance Li metal batteries. These mechanistic findings emphasize the need to understand electrolyte decomposition pathways to design tailored electrolyte formulations where each component can improve interfacial stability and ultimately enable smooth Li metal deposition in liquid electrolytes.

\section{Experimental}

Materials and Methods. Lithium metal ribbon ( $0.75 \mathrm{~mm}$ thick), dimethyl carbonate (DMC, anhydrous, $>99 \%$ ), and potassium bromide $(\mathrm{KBr}, 99 \%)$, were purchased from Sigma Aldrich. Bis(2,2,2trifluoroethyl)ether (BTFE, 99\%) was purchased from Synquest Laboratories. Lithium bis(fluorosulfonyl imide) (LiFSI, $>98.0 \%$ ) was purchased from Tokyo Chemical Industry. Dimethyl sulfoxide- $\mathrm{d}_{6}$ (DMSO- $\mathrm{d}_{6}$, 99.9\%) was purchased from Cambridge Isotope Laboratories. DMSO- $\mathrm{d}_{6}$ and BTFE were dried under molecular sieves for $48 \mathrm{~h}$ in an Ar-filled glovebox $\left(<0.1 \mathrm{ppm} \mathrm{O}_{2},<0.5 \mathrm{ppm} \mathrm{H}_{2} \mathrm{O}\right)$ and BTFE was filtered using a $200 \mathrm{~nm}$ pore size polytetrafluoroethylene (PTFE) syringe filter prior to use. $\mathrm{KBr}$ was dried in vacuo for a week at $100{ }^{\circ} \mathrm{C}$ before bringing into an Ar-filled glovebox. LiFSI was dried in vacuo for $24 \mathrm{~h}$ at 100 ${ }^{\circ} \mathrm{C}$ before bringing into the glovebox. Fluorinated ethylene propylene (FEP) NMR tube liners for operando NMR experiments were purchased from Wilmad Labglass. Low-pass rf filters, double-shielded electrochemistry wires, nonmagnetic wire clip, and rf shield were purchased from NMR Service.

Electrolyte formulations. Electrolytes of different compositions (1.2 M LiFSI in DMC, low concentration electrolyte, LCE; 1.2 M LiFSI in 1:2 DMC:BTFE by mole, localized high concentration electrolyte, LHCE) were prepared in an Ar-filled glovebox. The LCE showed 28 ppm $\mathrm{H}_{2} \mathrm{O}$, while the LHCE showed $10 \mathrm{ppm}$ $\mathrm{H}_{2} \mathrm{O}$ in Karl Fischer titration. 
Coulombic efficiency. Coulombic efficiency (CE) was measured using $\mathrm{Li} / \mathrm{Cu}$ coin cells at a current density of $0.5 \mathrm{~mA} \mathrm{~cm}^{-2} .0 .5 \mathrm{mAh} \mathrm{cm}^{-2}$ was deposited from the Li electrode onto the $\mathrm{Cu}$ substrate and then stripped for 100 cycles, with the stripping capacity:plating capacity ratio used to calculate $\mathrm{CE}$ for each cycle. Average CE and error bars representing standard error over three cells are plotted as a function of cycle number.

Cyclic voltammetry. Cyclic voltammetry was measured using Li/stainless steel coin cells, sweeping from open-circuit voltage to $2 \mathrm{~V}$, then down to $0 \mathrm{~V}$, and repeating for five cycles. The sweep rate used was $0.1 \mathrm{mV} / \mathrm{s}$.

Solution NMR measurements. Electrolyte samples were prepared by assembling Li/Li symmetric coin cells with glass fiber separators. For each electrolyte composition, at least two independent battery cells were analyzed and compared. Electrolyte was then immediately extracted for pristine samples, whereas cycled samples were prepared by cycling the coin cell at $1 \mathrm{~mA} \mathrm{~cm}^{-2}$ and $2 \mathrm{~h}$ charge/discharge cycles. Electrolyte was extracted by dipping Li electrodes and separator into $1.2 \mathrm{~mL} \mathrm{DMSO}-\mathrm{d}_{6}$, and compressing the separator using clean tweezers to allow the electrolyte to dissolve in the DMSO- $\mathrm{d}_{6}$. Electrolyte in DMSO- $\mathrm{d}_{6}$ solution was then filtered using a $200 \mathrm{~nm}$ pore size polytetrafluoroethylene (PTFE) syringe filter to remove Li and glass fiber separator. Filtered samples were then sealed in $5 \mathrm{~mm}$ air-tight $\mathrm{J}$ Young tubes in the glovebox for data acquisition.

All solution NMR experiments were performed on a Bruker Avance III 400 spectrometer equipped with a triple resonance broadband observe (TBO) probehead. All spectra were recorded at $\mathrm{T}=300 \mathrm{~K}$. Onedimensional (1D) ${ }^{1} \mathrm{H}\left(30^{\circ}\right.$ single pulse, $1 \mathrm{~s}$ recycle delay, 64 scans, internally referenced to residual DMSO at $2.5 \mathrm{ppm}),{ }^{7} \mathrm{Li}\left(90^{\circ}\right.$ single pulse, $2 \mathrm{~s}$ recycle delay, 8 scans, internally referenced to LiFSI at $\left.-1.04 \mathrm{ppm}\right)$, ${ }^{13} \mathrm{C}\left(30^{\circ}\right.$ single pulse with WALTZ-16 ${ }^{1} \mathrm{H}$ decoupling, $3 \mathrm{~s}$ recycle delay, 6144 scans, internally referenced to DMSO at $39.5 \mathrm{ppm})$ and ${ }^{19} \mathrm{~F}\left(30^{\circ}\right.$ single pulse, $2 \mathrm{~s}$ recycle delay, 32 scans, internally referenced to $\mathrm{FSI}^{-}$ at $51.2 \mathrm{ppm}$ ) NMR spectra were recorded on pristine electrolyte samples as well as electrolyte extracted from $\mathrm{Li} / \mathrm{Li}$ coin cells after cycling.

${ }^{7} \mathrm{Li}$ and ${ }^{19} \mathrm{~F}$ PFG-NMR spectra were collected using a bipolar gradient pulse sequence with gradient strengths varied from 2.5 to $47.5 \mathrm{G} \mathrm{cm}^{-1}$ in 16 increments. For ${ }^{7} \mathrm{Li}$, the gradient pulse duration $(\delta)$ was $4 \mathrm{~ms}$ and the time interval between gradient pulses $(\Delta$, the time during which species were allowed to diffuse during the experiment) was $200 \mathrm{~ms}$. For ${ }^{19} \mathrm{~F}, \delta=3 \mathrm{~ms}$ and $\Delta=100 \mathrm{~ms}$. NMR signal intensities were fit to the Stejskal-Tanner equation to obtain diffusion coefficients:

$$
I=I_{0} e^{-D \gamma^{2} G^{2} \delta^{2}\left(\Delta-\frac{\delta}{3}\right)}
$$

where $I_{0}$ is the unweighted signal intensity, $I$ is the signal intensity with the gradient applied, $\gamma$ is the gyromagnetic ratio of the observed nucleus, $G$ is the gradient strength, and $D$ is the diffusion coefficient.

A detailed discussion of operando NMR methods may be found in Figures S1 and S2. One dimensional operando ${ }^{19} \mathrm{~F}$ NMR measurements were performed using a $30^{\circ}$ single pulse, $5 \mathrm{~s}$ recycle delay, and 32 scans per experiment. Simultaneous CE measurement was performed at $2 \mathrm{~mA} \mathrm{~cm}^{-2}$, with 30 minutes of Li plating on $\mathrm{Cu}$ followed by stripping to $1 \mathrm{~V}$.

Solid-state NMR measurements. Individual samples were prepared by cycling $2032 \mathrm{Li} / \mathrm{Li}$ symmetric cells with Celgard 2325 separators at $1 \mathrm{~mA} \mathrm{~cm}^{-2}$ for $2 \mathrm{~h}$ charge/discharge cycles. After cycling, microstructural Li was removed from the electrode surface using a razor blade, and dried in vacuo overnight in a glass vial. Microstructural Li was mixed thoroughly with $\mathrm{KBr}(5: 1 \mathrm{KBr}: \mathrm{Li} \mathrm{w} / \mathrm{w})$ using a mortar and pestle in the glovebox to limit electrical connectivity of conductive Li particles and reduce eddy currents ${ }^{30}$ while 
spinning the sample in the NMR magnet before packing into a $1.6 \mathrm{~mm}$ o.d. $\mathrm{ZrO}_{2}$ rotor. Before loading into the SSNMR probehead, filled rotors were weighed to allow for quantitative comparison of the resulting SSNMR spectra.

All SSNMR experiments were performed on a Bruker Avance NEO $600 \mathrm{MHz}$ spectrometer equipped with a 1.6 mm HFXY MAS Phoenix NMR probehead. All spectra were collected at room temperature. Prior to each set of measurements, the magic-angle was set using $\mathrm{KBr} .{ }^{1} \mathrm{H}-{ }^{13} \mathrm{C}$ cross polarization magic-angle spinning (CPMAS) measurements were performed with a Hartmann-Hahn match of $60 \mathrm{kHz}$ with a ramped (90-100\%) pulse on ${ }^{1} \mathrm{H}$, a contact time of $2 \mathrm{~ms}$, a recycle delay of $5 \mathrm{~s}$, between 25840 and 30207 scans, and ${ }^{1} \mathrm{H}$ TPPM decoupling at $50 \mathrm{kHz}$ for the LCE sample and $90 \mathrm{kHz}$ for the LHCE sample. ${ }^{19} \mathrm{~F}$ SSNMR experiments were performed using a spin-echo pulse sequence $(\tau=167 \mu \mathrm{s})$, a $90^{\circ}$ pulse length of $3 \mu \mathrm{s}$, a recycle delay of $10 \mathrm{~s}$, and between 1243 and 7750 scans. ${ }^{7} \mathrm{Li}$ SSNMR experiments were performed using a single pulse acquisition with a $90^{\circ}$ pulse length of $2.62 \mu \mathrm{s}$, a recycle delay of $5 \mathrm{~s}$, and 64 scans. ${ }^{1} \mathrm{H}$ SSNMR experiments were performed using a spin-echo pulse sequence $(\tau=4.3 \mu \mathrm{s})$ with a $90^{\circ}$ pulse length of $2.15 \mu \mathrm{s}$, a recycle delay of $5 \mathrm{~s}$, and 64 scans. ${ }^{1} \mathrm{H}$ NMR was externally referenced to ${ }^{13} \mathrm{C}$ NMR of histidine at $183.3 \mathrm{ppm}$ and converted to other observe nuclei using the gyromagnetic ratios of ${ }^{13} \mathrm{C}$ and ${ }^{1} \mathrm{H}$. ${ }^{7} \mathrm{Li} \mathrm{NMR}$ was externally referenced to $\mathrm{LiF}$ at $-1 \mathrm{ppm}$, and ${ }^{19} \mathrm{~F}$ was externally referenced to $\mathrm{LiF}$ at -204 ppm.

Peak Fitting and Modelling. Python package nmrglue ${ }^{34}$ was used to extract processed ${ }^{17} \mathrm{O}$ NMR data from Bruker Topspin 3.6.1 files. Peaks were deconvoluted and fit to Lorentzian lineshapes using a least-squares minimization algorithm with Python library lmfit. ${ }^{36}$

X-ray photoelectron spectroscopy. $\mathrm{Li} / \mathrm{Li}$ symmetric coin cells were galvanostatically cycled using Celgard separators at $1 \mathrm{~mA} \mathrm{~cm}^{-2}$ for $2 \mathrm{~h}$ charge/discharge cycles. Li electrodes were removed from the cell and triple-washed in DMC (dipped $30 \mathrm{~s}$ per wash) to remove residual salts and prevent charging in the XPS. Samples were dried in vacuo overnight to remove residual electrolyte solvent and prevent sample offgassing in the evacuated XPS chamber. The samples were mounted on XPS stubs inside of the glovebox using carbon tape. Samples were transferred to the XPS sample chamber using an airtight Ar-filled jar, with exposure to atmosphere estimated to be $<5 \mathrm{~s}$ for each sample. Spectra were collected using a PHI 5600 XPS system with a hemispherical analyzer and an Al X-ray source with XPS base chamber pressure $<3.0 \times 10^{-}$ ${ }^{8}$ Torr. XPS Peak 41 software was used to fit spectra, providing both peak locations and integrations. The adventitious carbon peak in the $\mathrm{C} 1 \mathrm{~s}$ spectrum was referenced to $284.8 \mathrm{eV}$. Peaks were assigned to PEO (C 1s, 286-287 eV), $\mathrm{C}=\mathrm{O}(\mathrm{C} 1 \mathrm{~s}, \sim 289 \mathrm{eV}), \mathrm{LiF}(\mathrm{F} 1 \mathrm{~s}, \sim 685-686 \mathrm{eV})$, LiFSI (F 1s, 688-689 eV; N 1s, 399$400 \mathrm{eV}), \mathrm{NO}_{3}(\sim 404-405 \mathrm{eV}), \mathrm{NO}_{2}(\sim 407-408 \mathrm{eV}), \mathrm{Li}_{2} \mathrm{~S}(\sim 159-160 \mathrm{eV}), \mathrm{SO}_{3}(\sim 166-167 \mathrm{eV})$, and $\mathrm{SO}_{4}$ $(\sim 169-170 \mathrm{eV}) .{ }^{37}$ All peaks were fit using a Shirley baseline correction, with two constraints: i) the Gaussian:Lorentzian ratio was the same for all peaks in a given orbital, and ii) the fwhm was the same for all peaks in a given orbital.

Scanning electron microscopy. Scanning electron micrographs of the Li metal anode surface after galvanostatic polarization at $1 \mathrm{~mA} \mathrm{~cm}{ }^{-2}$ for $2 \mathrm{~h}$ were taken using a Zeiss Sigma VP Schottky Thermal Field emission SEM with a Gemini objective lens. Before imaging, all samples were double-washed in DMC (30 $\mathrm{s}$ each wash) to remove residual salts and prevent charging under the electron beam. Samples were dried in vacuo overnight to remove residual electrolyte and solvent. The samples were mounted on SEM stubs inside of the glovebox using carbon tape. Samples were transferred to the SEM sample chamber using an airtight Ar-filled jar, with exposure to atmosphere estimated to be $<5 \mathrm{~s}$ for each sample. 
Viscosity. Kinematic viscosities of electrolytes and electrolyte solvents (Figure S3) were measured manually in an Ar-filled glovebox using a U-tube viscometer. Five repetitions were performed for each composition, with standard error $<1 \%$ for all measurements. Densities of each electrolyte were measured using a micropipette and a balance to convert to dynamic viscosity.

\section{Author Contributions}

Conceptualization, R.M. and L.E.M.; Methodology, R.M., J.C.H., and L.E.M.; Investigation, R.M.; Writing - Original Draft, R.M. and L.E.M.; Writing—Review \& Editing, R.M., J.C.H., and L.E.M.; Supervision, L.E.M.; Funding Acquisition, L.E.M.

\section{Conflicts of interest}

There are no conflicts to declare.

\section{Acknowledgments}

This work was supported by National Science Foundation CAREER Award (CBET-2045262). Richard May is supported by the U.S. Department of Defense through the National Defense Science \& Engineering Graduate (NDSEG) Fellowship program. Julia C. Hestenes is supported by the National Science Foundation through the Graduate Research Fellowship Program (NSF GRFP).

\section{References}

1 E. Peled and S. Menkin, J. Electrochem. Soc., 2017, 164, A1703.

2 X. B. Cheng, R. Zhang, C. Z. Zhao, F. Wei, J. G. Zhang and Q. Zhang, Adv. Sci., 2015, 3, 1-20.

3 D. Aurbach, J. Power Sources, 2000, 89, 206-218.

4 A. J. Ilott and A. Jerschow, J. Phys. Chem. C, 2018, 122, 12598-12604.

$5 \quad$ C. Fang, X. Wang and Y. S. Meng, Trends Chem., 2019, 1, 152-158.

6 O. Borodin, J. Self, K. A. Persson, C. Wang and K. Xu, Joule, 2020, 4, 69-100.

$7 \quad$ Y. Yamada and A. Yamada, J. Electrochem. Soc., 2015, 162, A2406.

8 S. K. Jeong, H. Y. Seo, D. H. Kim, H. K. Han, J. G. Kim, Y. B. Lee, Y. Iriyama, T. Abe and Z. Ogumi, Electrochem. commun., 2008, 10, 635-638.

9 L. Suo, O. Borodin, T. Gao, M. Olguin, J. Ho, X. Fan, C. Luo, C. Wang and K. Xu, Science, 2015, 350, 938-943.

10 C. A. Angell, C. Liu and E. Sanchez, Nat. 1993 3626416, 1993, 362, 137-139.

11 Y. Yamada, K. Furukawa, K. Sodeyama, K. Kikuchi, M. Yaegashi, Y. Tateyama and A. Yamada, 
J. Am. Chem. Soc., 2014, 136, 5039-5046.

12 M. Winter, B. Barnett and K. Xu, Chem. Rev., 2018, 118, 11433-11456.

13 J. B. Goodenough and Y. Kim, Chem. Mater., 2010, 22, 587-603.

14 Y. Zheng, F. A. Soto, V. Ponce, J. M. Seminario, X. Cao, J. G. Zhang and P. B. Balbuena, J. Mater. Chem. A, 2019, 7, 25047-25055.

15 Y. Zheng and P. B. Balbuena, J. Chem. Phys., 2021, 154, 104702.

16 J. Qian, W. A. Henderson, W. Xu, P. Bhattacharya, M. Engelhard, O. Borodin and J. G. Zhang, Nat. Commun., 2015, 6, 6362.

17 X. Ren, S. Chen, H. Lee, D. Mei, M. H. Engelhard, S. D. Burton, W. Zhao, J. Zheng, Q. Li, M. S. Ding, M. Schroeder, J. Alvarado, K. Xu, Y. S. Meng, J. Liu, J. G. Zhang and W. Xu, Chem, 2018, 4, 1-16.

18 S. Chen, J. Zheng, D. Mei, K. S. Han, M. H. Engelhard, W. Zhao, W. Xu, J. Liu and J. G. Zhang, Adv. Mater., 2018, 30, 1706102.

19 X. Cao, H. Jia, W. Xu and J.-G. Zhang, J. Electrochem. Soc., 2021, 168, 010522.

20 X. Ren, L. Zou, X. Cao, M. H. Engelhard, W. Liu, S. D. Burton, H. Lee, C. Niu, B. E. Matthews, Z. Zhu, C. Wang, B. W. Arey, J. Xiao, J. Liu, J. G. Zhang and W. Xu, Joule, 2019, 3, 1662-1676.

21 D. Aurbach, M. Daroux, P. Faguy and E. Yeager, J. Electroanal. Chem., 1991, 297, 225-244.

22 X. Zhang, R. Kostecki, T. J. Richardson, J. K. Pugh and P. N. Ross, J. Electrochem. Soc., 2001, 148, A1341.

23 L. L. Jiang, C. Yan, Y. X. Yao, W. Cai, J. Q. Huang and Q. Zhang, Angew. Chemie - Int. Ed., 2021, 60, 3402-3406.

24 X. Bogle, R. Vazquez, S. Greenbaum, A. V. W. Cresce and K. Xu, J. Phys. Chem. Lett., , DOI:10.1021/jz400661k.

25 L. E. Camacho-Forero and P. B. Balbuena, Phys. Chem. Chem. Phys., 2017, 19, 30861-30873.

26 L. F. Nazar, M. Cuisinier and Q. Pang, MRS Bull. 2014 395, 2014, 39, 436-442.

27 A. Manthiram, Y. Fu, S.-H. Chung, C. Zu and Y.-S. Su, Chem. Rev., 2014, 114, 11751-11787.

28 R. May, K. J. Fritzsching, D. Livitz, S. R. Denny and L. E. Marbella, ACS Energy Lett., 2021, 6, 1162-1169.

29 R. May, Y. Zhang, S. R. Denny, V. Viswanathan and L. E. Marbella, Cell Reports Phys. Sci., 2020, 1, 100239.

30 N. Leifer, M. C. Smart, G. K. S. Prakash, L. Gonzalez, L. Sanchez, K. A. Smith, P. Bhalla, C. P. Grey and S. G. Greenbaum, J. Electrochem. Soc., 2011, 158, A471-A480.

31 M. Leskes, G. Kim, T. Liu, A. L. Michan, F. Aussenac, P. Dorffer, S. Paul and C. P. Grey, J. Phys. Chem. Lett., 2017, 8, 1078-1085.

32 V. Etacheri, O. Haik, Y. Goffer, G. A. Roberts, I. C. Stefan, R. Fasching and D. Aurbach, Langmuir, 2012, 28, 965-976.

33 Y. Jin, N. J. H. Kneusels, P. C. M. M. M. M. Magusin, G. Kim, E. Castillo-Martínez, L. E. 
Marbella, R. N. Kerber, D. J. Howe, S. Paul, T. Liu and C. P. Grey, J. Am. Chem. Soc., 2017, 139, 14992-15004.

34 S. Jurng, Z. L. Brown, J. Kim and B. L. Lucht, Energy Environ. Sci., 2018, 11, 2600-2608.

35 Q. Zhao, Z. Tu, S. Wei, K. Zhang, S. Choudhury, X. Liu and L. A. Archer, Angew. Chemie - Int. Ed., 2018, 130, 1004-1008.

36 W. Xue, Z. Shi, M. Huang, S. Feng, C. Wang, F. Wang, J. Lopez, B. Qiao, G. Xu, W. Zhang, Y. Dong, R. Gao, Y. Shao-Horn, J. A. Johnson and J. Li, Energy Environ. Sci., 2020, 13, 212-220.

37 D. Aurbach, E. Pollak, R. Elazari, G. Salitra, C. S. Kelley and J. Affinito, J. Electrochem. Soc., 2009, 156, A694-A702. 